\title{
Analysis of Inhomogeneities in Hydrogen Storage Alloys: A Comparison of Different Methods
}

\author{
Francesco Massimino ${ }^{1,2}$ \\ ${ }^{1}$ Università degli Studi, Dipartimento di Chimica IFM, Turin, Italy \\ ${ }^{2}$ NTT_-New Tera Technology, Turin, Italy \\ Email: massimino.francesco@gmail.com
}

Received October 26, 2012; revised November 22, 2012; accepted November 30, 2012

\begin{abstract}
In this work we have realized a simplified model to analyze compositional inhomogeneities in commercial hydrogen storage alloys. We have used it to evaluate the effect of the thermal annealing, together with calorimetric, PCIs and XRD measurement. We have compared results with composition distribution histograms based on Rietveld refinement of XRD patterns. Finally we studied the variation of $\alpha$ and $\beta$ phases crystallographic parameters with $\mathrm{H} 2$ pressure.
\end{abstract}

Keywords: Hydrogen Storage; Alloys; Inhomogeneities; Sloping Plateau

\section{Introduction}

Pressure-Composition Isotherms (PCIs) are one of the most informative data when performances of hydrogen storage alloys need to be determined. Basically these curves should be composed, at least for simple systems, of three branches: a stiff rise at the beginning, a flat and wide plateau central region and a second stiff rise at the end. The first and the last part are related to hydrogen solubility, respectively in metal and in hydride. The central flat part is due to transition of metal into hydride phase: as every two-phase equilibrium at a certain temperature it should lay at one well-defined pressure. It is common to obtain a sloping plateau instead of a flat one. Many hypotheses have been proposed to explain it, including kinetic effects and establishment of local equilibriums [1], microstructure and internal stresses [2] and inhomogeneities of the alloy: Park et al. [3] proposed to use this feature of the PCIs as a diagnostic method for inhomogeneities.

In the following we tried to implement a simplified and easily applicable version of this model in the analysis of a commercial $\mathrm{LaNi}_{4.8} \mathrm{Al}_{0.2}$ alloy and we compared results with the ones we obtained from other adhoc experiments based on Rietveld refinement of XRD pattern of both metal powder and partially hydrided powder.

\section{Experimental}

Experiments have been carried on $\mathrm{LaNi}_{4.8} \mathrm{Al}_{0.2}$ from Palcan Energy Corporation and 5.0 grade hydrogen has been used.

As-received alloy has been observed in SEM and EDS microanalysis has been used to determine coarsely the average composition of the alloy.

Part of the alloy has been annealed in Ar for one week at $1000^{\circ} \mathrm{C}$.

$\mathrm{XRD}$ patterns of as-received and annealed alloys have been collected both in vacuum and at different hydrogen pressures, PCIs and calorimetric curves have been measured (see below for further details of each part).

Alloys have been activated following this general procedure for hydrogen applications:

- Preliminary coarse grinding;

- In-vacuum heating to $100^{\circ} \mathrm{C}$;

- Soak in high hydrogen pressure (15 - 50 bar depending on the apparatus used);

- Cooling down to room temperature after one hour.

This procedure has been repeated twice after each air exposure of samples.

\subsection{Pressure-Composition Curves}

PCIs curves have been measured with two different apparatus.

The first one is an automatic Sievert AMC Unit, used to trace PCI curves at temperatures in the range of $25^{\circ} \mathrm{C}$ $120^{\circ} \mathrm{C}$. The instrument is equipped with an oven and a temperature controller and provides corrections for $\Delta \mathrm{T}$ between hydrogen reservoir and specimen region. Hydrogen sorbed amounts are calculated using modified Benedict-Webb-Rubin equation for real gases.

The second apparatus is a simplified version of the first one: it consists of a manually operated high pressure steel line, in which ideal gas law is used to evaluate hy- 
drogen sorbed amount. In this case there is no $\Delta \mathrm{T}$ correction: it is accurate for measurement at room temperature, but it tends to overestimate/underestimate sorbed amounts at temperatures lower/higher than room temperature. The same apparatus is equipped with a C80 calorimeter that allows to measure reaction enthalpies. Pressure is measured with a capacitive transducer.

Reaction enthalpies and entropies can also be estimated from Van't Hoff equation, mathematically operating on PCI curves at different temperatures.

\subsection{C80 Calorimeter}

Setaram C80 DTA calorimeter is used together with the steel high pressure line previously mentioned. The reference cell is identical to the specimen one; the two cells are connected, so they undergo the same pressure conditions. Signal has been amplified and collected through a LabView custom interface. All measures have been done at constant temperature, thanks to a heater and a temperature controller.

A considerable quantity of sample (6 g) has been used in experiment: in this way it was possible to observe good $\mathrm{S} / \mathrm{N}$ ratio in the thermal signal and to minimize error in determining sorbed amount, without changing too much hydrogen concentration in the material. In order to improve reproducibility and standardization of the measurement, a fixed cut-off has been introduced in the thermal peak: this avoids as much as possible the effect of thermal oscillation but it may introduce some error, especially in measurements in the plateau region, that is very temperature-dependent; the recovery of the initial temperature may take a really long time due to thermal ballast action operated by hydrides: cut-off may eliminate part of the signal, but at least this error is systematic for all measurement.

Ratio between the integral of thermal peak and the sorbed quantity of hydrogen, that represents enthalpy of reaction, is converging with time and someway reduces the negative effect of the introduction of the cut-off.

Empty cell tests confirmed that heat involved with gas expansion in and from the cell region is negligible in those experiments.

Generally each part of the specimen is considered as it has sorbed the same hydrogen amount at the end of each sorption step; this fact may not be true: due to hysteresis phenomena and really quick reaction rate, for instance, upper part of the sample may absorb more than the lower one and not returning hydrogen at the considered pressure, causing non-homogeneities along the vertical axis.

\subsection{X-Ray Diffraction}

XRD has been performed with a Phillips X'pert in Bragg-Brentano configuration, equipped with plate sam- ple-holder or XRK900 reactive stage, with $\mathrm{Cu}$ X-ray tube; XRK900 has Be windows, Ni filter has been removed in this case to maximize signal and improve acquisition speed.

The sample region is linked to a high pressure and vacuum line that allows imposing a specific hydrogen pressure. Pressure is measured with a capacitive transducer; we have estimated a pressure drop between transducer and cell of 0.5 bar in the 1 - 3 bar region due to resistive effect of the pipes.

We have performed XRD at different fractions of the plateau region. Measures have been executed at the same temperature of the calorimetric one.

For each sorption step several XRD pattern has been collected quickly $\left(0.75^{\circ} / \mathrm{min}\right)$ and consequently in a short degree range $\left(2 \theta=38^{\circ} \sim 44^{\circ}\right)$ containing the two major peaks of $\alpha$ and $\beta$ phases; when XRD pattern was not changing any more, a large degree range $\left(2 \theta=25^{\circ} \sim 75^{\circ}\right)$ XRD patterns have been collected, also with longer acquisition time $\left(0.33^{\circ} / \mathrm{min}\right)$.

With plate sample-holder we averaged four acquisitions in an even wider range $\left(2 \theta=20^{\circ} \sim 100^{\circ}\right)$ with longer acquisition time $\left(0.33^{\circ} / \mathrm{min}\right)$.

Rietveld analysis has been accomplished using MAUD software [4] on large XRD patterns. Instrumental broadening function has been built using standard LaB6. Due to the removal of $\mathrm{Ni}$ filter, also $\mathrm{k} \beta$ has been introduced in the Rietveld simulation for XRD pattern acquired with reactive chamber stage.

First we have refined Rietveld simulation of a single phase for in vacuum XRD pattern and from this step we have obtain single phase cell parameters. Then we have introduced in the simulation more metal phases with slightly different Al contents. In the second case we have kept constant B factors and microstructure parameters in addiction to previous ones. Relative phase fraction has been achieved from the computation.

In the case of reactive chamber XRD patterns, Rietveld refinement has been performed introducing two phases, $\alpha$-solution and $\beta$-hydride; Al occupancy has been fixed to reach nominal stoichiometry of the alloy. Cell parameters and phase ratio have been obtained from the computation.

\section{Data Treatment and Calculation}

For the following calculations we needed functions describing $p_{p l}\left(x_{A l}\right), a\left(x_{A l}\right)$ and $c\left(x_{A l}\right)$ and where $p_{p l}$ is plateau pressure, $a$ and $c$ are cell parameters and $x_{A l}$ is the Al content. We have described $p_{p l}$ with Van't Hoff equation.

$$
\ln p_{p l}==-\frac{|\Delta H|}{R T}+\frac{|\Delta S|}{R}
$$

so we had also to determine $\Delta H\left(x_{A l}\right)$ and $\Delta S\left(x_{A l}\right)$. 
We data mined in literature [5-8] and in our previous lab measurements for these parameters and we elaborated linear expressions for desired functions. Available data allowed us to determine solidly $\Delta H\left(x_{A l}\right)$ and $\Delta S\left(x_{A l}\right)$ for absorption process only.

Linear parameters are shown in Table 1.

Low resolution PCIs, hydrogen $\mathrm{wt} \%$ vs. $\ln (p)$, have been fitted with a sigmoidal curve to better obtain their derivative curve: with this assumption we hypothesize gaussian distribution of inhomogeneities as Fujitani et al. [9] did.

The complete expression (2) Park et al. [3] proposed has been significantly simplified, once they have verified the distribution character of their function.

$$
f(\ln p)=\frac{\mathrm{d} \theta / \mathrm{d} \ln p^{-k(\theta)}}{\theta_{\beta}-\theta_{\alpha}}
$$

In this expression $\theta$ indicates $\mathrm{H} / \mathrm{M}$ atom ratio, $\alpha$ and $\beta$ are metal and hydride phase and $\theta_{\alpha}$ and $\theta_{\beta}$ are phase boundaries.

$$
\begin{aligned}
k(\theta) & =\frac{k_{b}\left(\theta-\theta_{\alpha}\right)+k_{a}\left(\theta_{\beta}-\theta\right)}{\theta_{\beta}-\theta_{\alpha}} \\
& =\frac{\theta\left(k_{b}-k_{a}\right)+k_{a} \theta_{\beta}-k_{b} \theta_{\alpha}}{\theta_{\beta}-\theta_{\alpha}}
\end{aligned}
$$

In Equation (3) $k_{b}$ and $k_{a}$ are the slopes of the $\theta(\ln p)$ PCI in first and last branch; because of the almost horizontality of these branch in our case the numerical value is very low and even more their difference that may be considered zero. $K(\theta)$ is almost constant and it lowers the value of $f(\ln p)$, amplifying the central values of the distribution and narrowing the function. If $K(\theta)$ is ne-glected the curve obtained may result broadened proportionally to the values of $k_{b}$ and $k_{a}$ in respect to the one obtained from the full calculation.

The denominator $\left(\theta_{\beta}-\theta_{\alpha}\right)$ assumes just a normalizing role and the derivative $\mathrm{d} \theta / \mathrm{d} \ln p$ remains the only fundamental factor; the normalization of the distribution can be imposed a posteriori at the end of the data treatment.

Note that the usage of $\mathrm{H} / \mathrm{M}$ atom ratio is no longer required and one can choose his preferred unit, e.g. hydrogen $\mathrm{wt} \%$.

The last step was converting $\mathrm{f}(\ln p)$ into $f\left(\mathrm{x}_{\mathrm{Al}}\right)$ using the Van't Hoff equation and linearized $\Delta H(x)$ and

Table 1. Cell and thermodinamic parameters vs. Al contents, linearized expressions.

\begin{tabular}{ccccc}
\hline & $a$ & $c$ & $\Delta H$ & $\Delta S$ \\
\hline Intercept & $5.0175(8)$ & $3.976(3)$ & $-31.10(6)$ & $-109.0(1.6)$ \\
Slope & $0.0362(6)$ & $0.0889(6)$ & $16.6(4)$ & $-9.7(3)$ \\
\hline
\end{tabular}

$\Delta S(x)$ expressions.

For what concerns the calorimetric curve, we have defined an average weight percent to avoid problems cited at the end of C80 paragraph: we have associated each measured $\Delta H$ to the mean value between current hydrogen $\mathrm{wt} \%$ and the one related to previous step, that is lower for absorption and higher for desorption.

In the multi-phase Rietveld refinement we have avoided to simulate the presence of too many phases, this would have introduced too many degree of freedom. We have used a maximum of five phases, fixed in Al content and cell parameters; they have been "prepared" using the linearized function for $a(x)$ and $c(x)$. The phases differ by a constant $\Delta x_{A l}$ value and each one is representative of a class of the same width. We have repeated the refinement using a different sampling of the phase space, namely "preparing" other phases differing $\Delta x_{A l} / 2$ from each one of the previous. After that we averaged the partially superposed classes, obtaining a histogram composed of classes with half broadness.

Finally in order to build a distribution based on the reactive chamber experiments, we used hydride-metal phase ratio obtained from the Rietveld computations and we associated each increment in the hydride quantity to the reached pressure. Then we converted pressure into $x_{A l}$ as done in the previous. The classes obtained in this way are not of the same wideness and cannot be used to plot a distribution histogram: for that reason we have normalized the value of each class dividing it by the class wideness and we normalized the whole distribution a posteriori.

\section{Results}

SEM imaging could not highlight inhomogeneities both in the as-received and annealed alloy. Also EDS analysis variability stays widely inside experimental uncertainly and gives atomic composition of $\mathrm{La} 18.6 \%$, Ni $78.8 \%$ and $\mathrm{Al} 2.6 \%$, corresponding to an $\mathrm{Al} /(\mathrm{Al}+\mathrm{Ni}) * 5$ ratio of 0.16 , a bit lower than nominal one of 0.2 .

XRD peaks, as shown in Figure 1, broaden for as-received alloy: this is particularly evident for $(00 l)$ peaks. Sharper peaks may be an index of less strained microstructure, as expected after annealing.

PCIs plateau flatten after annealing; PCIs of as-received and annealed alloys cross exactly at midpoint of plateau and hysteresis remains of the same magnitude. In Figure 2 PCIs collected at $313 \mathrm{~K}$ with manual instrument for both alloys are shown. The flattening of the plateau after annealing is a clear clue suggesting the presence of inhomogeneities in the received alloy.

As shown in Figure 3, reaction enthalpy, determined with $\mathrm{C} 80$, is constant for the annealed alloy and it is about $33.8 \mathrm{~kJ} / \mathrm{mol}$, while the result for the as-received alloy is higher for first-absorbed (or last-desorbed) hy 


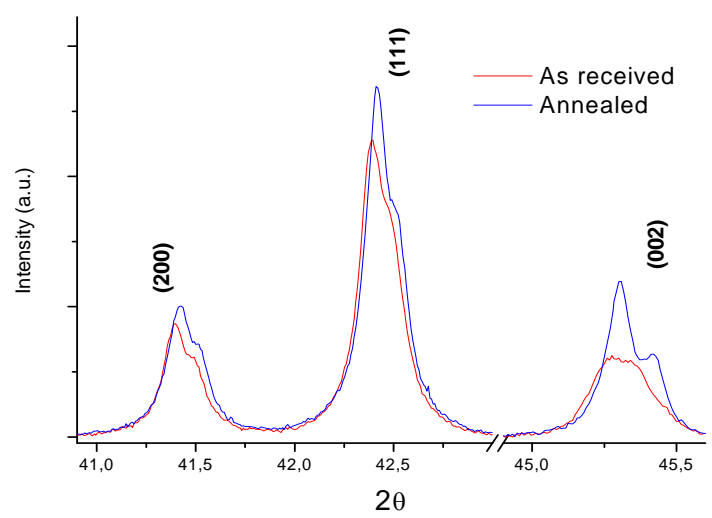

Figure 1. XRD peaks of as-received and annealed alloys.

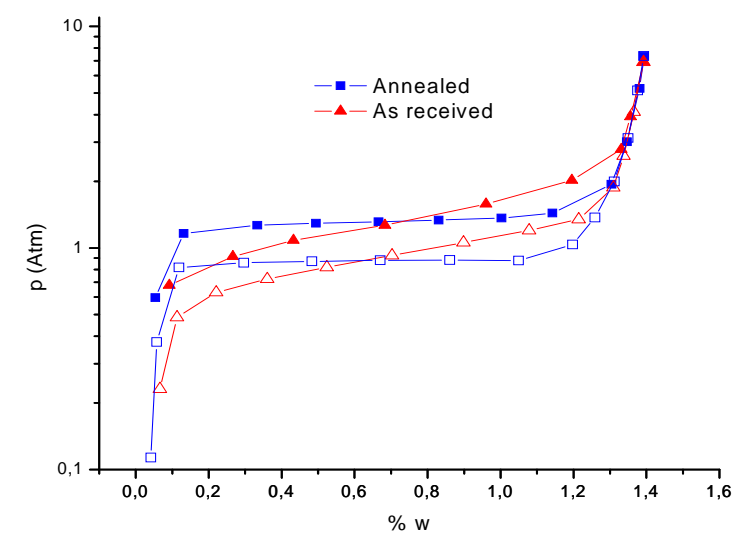

Figure 2. PCIs at $313 \mathrm{~K}$ of as-received and annealed alloys.

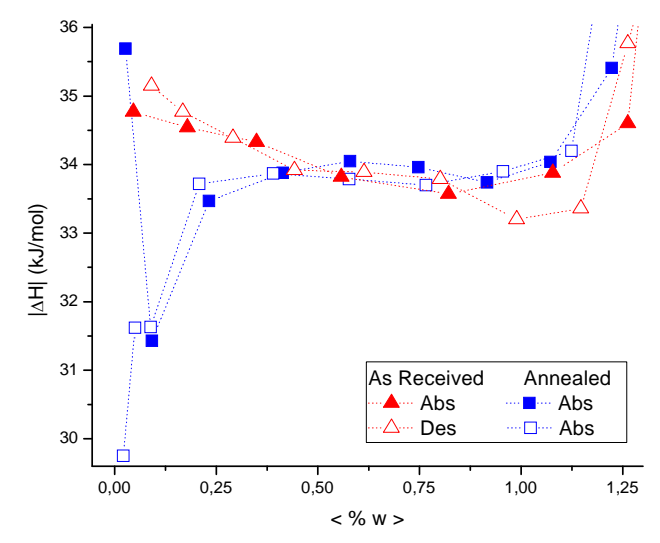

Figure 3. Calorimetric determined $|\Delta \mathrm{H}|$ at $313 \mathrm{~K}$ as-received and annealed alloys; this data have been collected contemporary to the PCIs of Figure 4.

drogen molecules and lower toward the final part of plateau. High heat of reaction is related to higher Al-content. Calorimetric curve is confirming that the modification of PCIs after annealing is not a merely kinetic effect.

Execution of the experiment with reactive chamber was greatly more problematic in the case of the annealed alloy: in fact the presence of a sloping plateau allowed us to explore more systematically the sample in the whole central range, while the flat plateau forces the alloy to pass abruptly from an almost pure $\alpha$ solid solution to a completely hydrided material $\beta$. For this reason there is a gathering of data to the sides of the plateau.

Figure 4 sums up the results of Rietveld refinement coming from this experiment.

Cell volume of the $\alpha$ phase suddenly increases after the first hydrogen load; $\alpha$ cell volume of as-received alloy shrinks with the rise of hydrogen pressure after the same initial expansion. In the case of $\beta$ phase, for as-received there is a gradual increment of volume, while in the annealed alloy it remains almost constant until a stiff rise in the last part.

The same trend can be directly and qualitatively observed from the XRD pattern; the main peaks of both phases are shown in Figure 5 that underlines also the high variability of (002) hydride peak in relation to the increasing $\mathrm{H}_{2}$ pressure.

Figure 6 presents the distributions and the histograms obtained from the various techniques previously described.

Every distribution shows a narrowing effect of the annealing process. Manual and automatic PCI instrument results are almost perfectly superposing, in particular for as-received sample. Centers of XRK and sloping plateau distributions coincide while the multi-phase Rietveld refinement gives results at substantially higher $x_{A l}$ values.

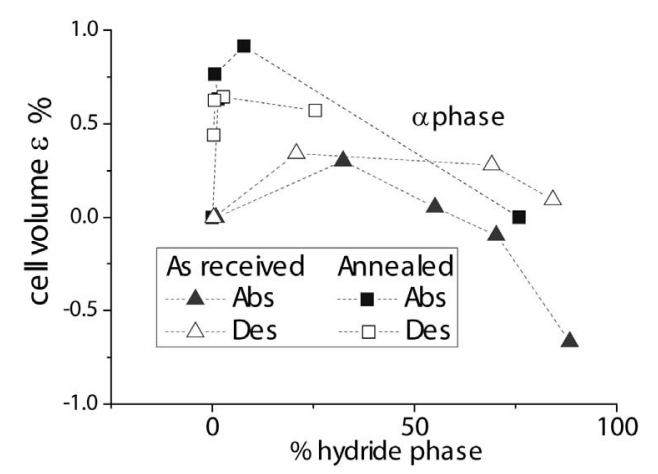

(a)

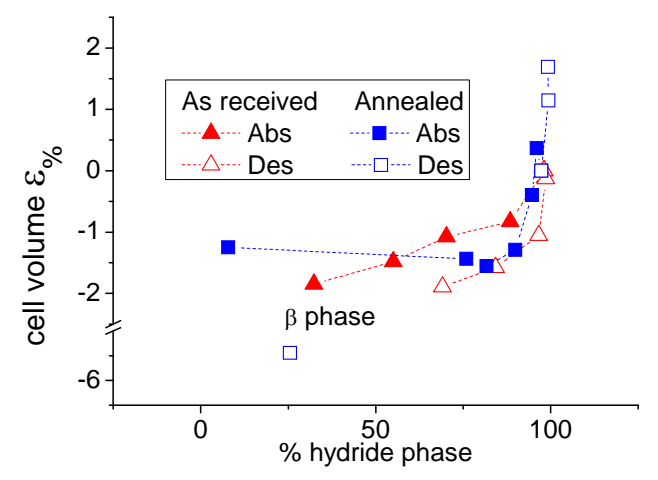

(b)

Figure 1. Cell volume variation: $\alpha$ phase take 0 bar phase as reference (a); $\beta$ phase take 10 bar phase as reference (b). 


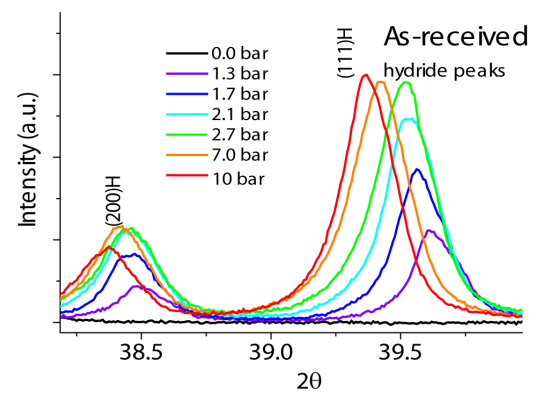

(a)

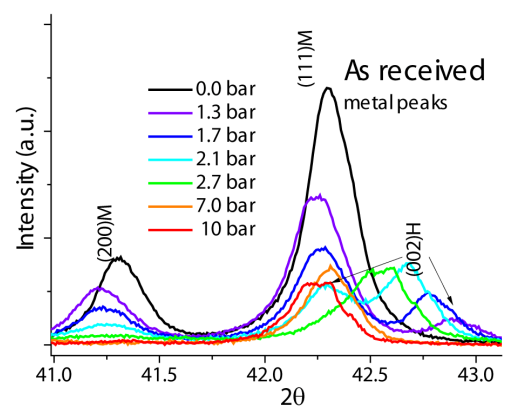

(b)

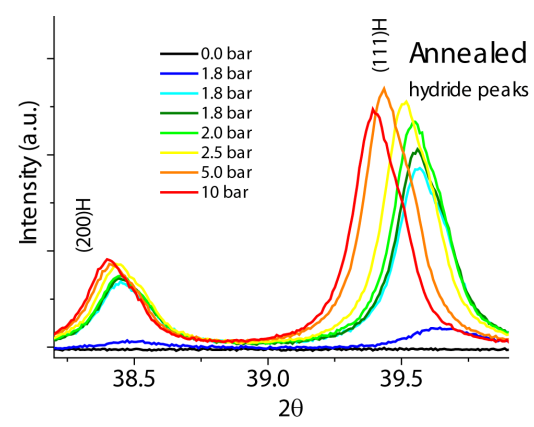

(c)

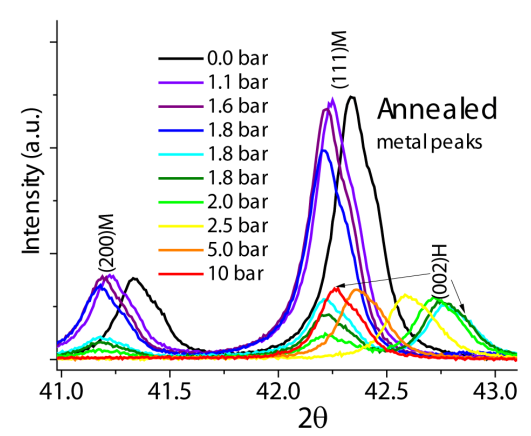

(d)

Figure 5. Main XRD peaks of $\alpha$ and $\beta$ phase during the reactive chamber experiment.

\section{Discussion}

Almost all the analyses performed on the bought alloy suggest that the material is not homogeneous; variations in $\mathrm{Al}$ content, and consequently on $\mathrm{Ni}$ one, change molecular weight of the unit formula of the alloy of 33 $\mathrm{g} / \mathrm{mol}$ per unitary $\Delta x_{A l}$ : even hypothesizing a variation of

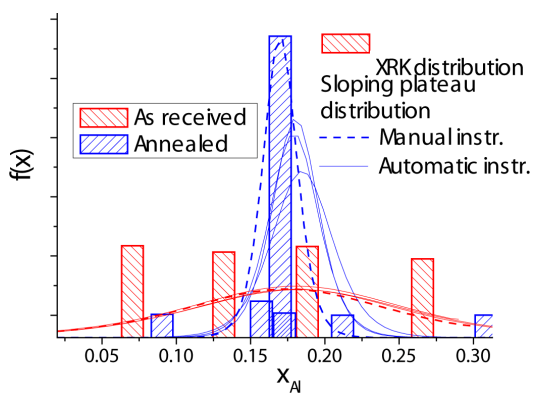

(a)

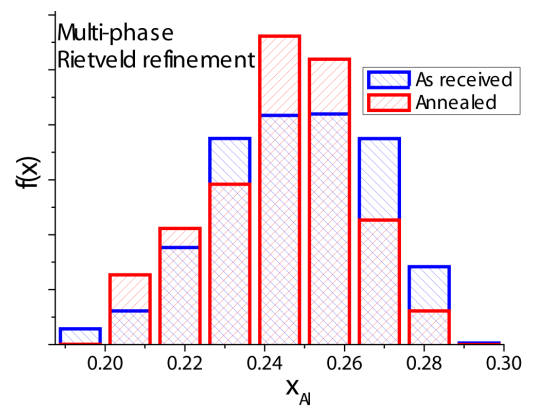

(b)

Figure 6. Distributions from various techniques: (a) histograms from reactive chamber experiment, lines from sloping plateau simplified model, dashed and continuous ones come from manual and automatic instrument data respectively; (b) histograms come from multi-phase Rietveld refinement.

0.3 on $x A_{l}$ it is not enough to appear with SEM imaging or to be clearly detected with EDS micro-analysis. For this reason we have no information about the spatial distribution of the inhomogeneities. Repeating of the PCIs measurement on specimen taken from other parts of the ingot brings to the same results: inhomogeneities are substantially equally spread all over the material.

The source of inhomogeneities is still not clear, but one hypothesis may be segregation acting on each grain during solidification from the melt.

$c$ cell parameter exhibits a greater dependency from both $x_{A l}$ and hydrogen concentration (i.e. pressure), as shown by the higher value of slope for linearized $c x_{A l}$ and shift of the $(00 l)$ peaks. Notice that percent variation of $c\left(x_{A l}\right)$ is even greater due to smaller dimension of $c$ compared to $a$. This relative stability of the $a$ parameter allows the growth of phases with slightly different $x_{A l}$ with relative low strain, at least in some direction. As a consequence XRD pattern is not so different after annealing, expecially for $(h k 0)$ peaks.

Calorimetric measurement confirmed the presence in the as-received alloy of Al-richer and Al-poorer fractions, fraction that tends to the average composition after the annealing: this fact is supported by the midpoint interception of PCIs collected both before and after annealing.

Trend of cell volume of $\alpha$ and $\beta$ is the result of three 
different phenomena:

1) $\mathrm{H}_{2}$ rising pressure increments the gas solubility in both $\alpha$ and $\beta$ phase, the higher is the hydrogen dissolved in a phase the bigger is its crystalline cell;

2) In inhomogeneous alloys Al-richer fractions are reacting at lower $\mathrm{H}_{2}$ pressure, and the more pressure rise, the more unreacted fraction is made of Al-poorer phases, which are the last to transform into $\beta$ phase; Al-poorer has smaller cell parameters, so the average $a$ and $c$ decrease in both $\alpha$ and $\beta$ phases, increasing $\mathrm{H}_{2}$ pressure for this effect;

3) As every material, this alloy may shrink due to increasing hydrostatic pressure and re-expand when pressure lowers; considering the low pressures involved this factor may be the less important, at least in the plateau region.

Observing cell volume $\varepsilon \%$ trends one may conclude that:

1) Effect is very strong for $\beta$ phase and really low, except for first hydrogen load, for $\alpha$ solid solution; this effect is basically the only one observed in the right absorption branch of annealed alloy; this difference of behavior may correspond to a dissimilarity in Sievert's constants;

2) Effect is present in as-received alloy for $\alpha$ phase but is overcome by $i$. effect for $\beta$ phase;

3) Effect may explain the increase of hydride cell volume as a relaxation while pressure is lowered after a complete hydrogen load.

There is agreement between sloping plateau model distributions and the ones based on reactive chamber experiments. In those cases the conversion between $\ln p$ and $x_{A l}$ is based on the same thermodynamic literature data $\Delta H$ and $\Delta S$. The automatic instrument curve of the annealed alloy is a bit broadened respect to the one coming from manual measurement: in the second apparatus the time between one step and the following is extremely longer than in the first one, due to the waiting needed to have the calorimeter correctly settled; for this reason the automatic measure may be influenced by some kinetic effects and same effects propagate to the distribution curve.

The multi-phase Rietveld distribution overestimates average $x_{A l}$. This problem seems to be intrinsic of the method. If one tries to simulate a single phase XRD over the same experimental data, obtained cell parameters correspond to a correct $x_{A l}(0.17$ in this case); once more phases have been introduced, the ones with bigger cell size gain in scale factor during the refinement and higher $\left\langle x_{A l}\right\rangle$ is obtained at the end of the process. Al content has not as its only effect the increase of the cell size, but it also changes relative heights of the XRD peaks in a nonlinear way. According to this statement the best agreement between experimental data and simulated curve may be found in different conditions in the singlephase refinement respect to the multi-phase one. Literature data come from a "single-phase library" and it may be not so correct to use it for a multi-phase simulation.

Considering this, the only information this multi-phase simulation is correctly furnishing is that there is an effective narrowing of the inhomogeneities distribution after annealing.

\section{Conclusions}

Commercial hydrogen storage alloys may have compositional inhomogeneities related to their production processes.

The simplified model we proposed seems to be a quick, good and simple way to estimate the variations in composition of one element $x$ in an alloy belonging to a system in which the function $p_{p l}(x)$ is known. A low resolution PCIs is sufficient for the whole calculation. This simplified model could be extended, with the knowledge of more functions (e.g. $\Delta H(x)$ and $\Delta S(x))$ and the availability of PCIs at different temperatures, to a more complex system with three or more varying atomic species.

Three effects are acting in the variation of the crystallographic parameters with $\mathrm{H}_{2}$ pressure and their trends may be very complex.

Multi-phase Rietveld refinement is probably not a fully trustable analytical technique with the available data for this kind of material; however it may be an interesting field into systematically inquire to build an alternative data library for future studies.

\section{REFERENCES}

[1] W. A. Oates and T. B. Flanagan, "On the Origin of Increasing Hydrogen Pressures in the Two Solid Phase Regions of Intermetallic Compound-Hydrogen Systems," Scripta Metallurgica, Vol. 17, No. 8, 1983, pp. 983-986. doi:10.1016/0036-9748(83)90435-0

[2] D. Wang, T. B. Flanagan and T. Kuji, "Hysteresis Scans for Pd-H and Pd-alloy-H Systems," Physical Chemistry Chemical Physics, Vol. 4, No. 17, 2002, pp. 4244-4254. doi:10.1039/b201271p

[3] C. Park, "Analysis of Sloping Plateaux in Alloys and Intermetallic Hydrides I. Diagnostic Features," Journal of Alloys and Compounds, Vol. 384, No. 1-2, 2004, pp. 203207. doi:10.1016/j.jallcom.2004.04.101

[4] L. Lutterotti, "Maud-Materials Analysis Using Diffraction," 2011.

[5] C. Da-li, et al., "Effects of Al Partial Substitution for Ni on Properties of $\mathrm{LaNi}_{5-\mathrm{x}} \mathrm{Al}_{\mathrm{x}}$," Transactions of Nonferrous Metals Society of China, Vol. 17, No. S1, 2007, pp. s967s971.

[6] J. Liu, et al., "Electrochemical Characterization of $\mathrm{LaNi}_{5-\mathrm{x}} \mathrm{Al}_{\mathrm{x}}(\mathrm{x}=0.1-0.5)$ in the Absence of Additives," Journal of Power Sources, Vol. 161, No. 2, 2006, pp. 
1435-1442. doi:10.1016/j.jpowsour.2006.05.037

[7] T. Kodama, "The Thermodynamic Parameters for the $\mathrm{LaNi}_{5-\mathrm{x}} \mathrm{Al}_{\mathrm{x}}-\mathrm{H}_{2}$ and $\mathrm{MmNi}_{5-\mathrm{x}} \mathrm{Al}_{\mathrm{x}}-\mathrm{H}_{2}$ Systems," Journal of Alloys and Compounds, Vol. 289, No. 1-2, 1999, pp. 207212. doi:10.1016/S0925-8388(99)00173-5

[8] H. Senoh, et al., "Systematic Investigation on Hydrogen Storage Properties of $\mathrm{RNi}_{5}$ (R: Rare Earth) Intermetallic Compounds with Multi-Plateau," Materials Science and
Engineering B, Vol. 108, No. 1-2, 2004, pp. 96-99. doi:10.1016/j.mseb.2003.10.055

[9] S. Fujitani, et al., "Development of Hydrogen-Absorbing Rare-Earth Ni-Alloys for a 20-Degrees-C Refrigeration System," Journal of Alloys and Compounds, Vol. 192, No. 1-2, 1993, pp. 170-172.

doi:10.1016/0925-8388(93)90221-8 